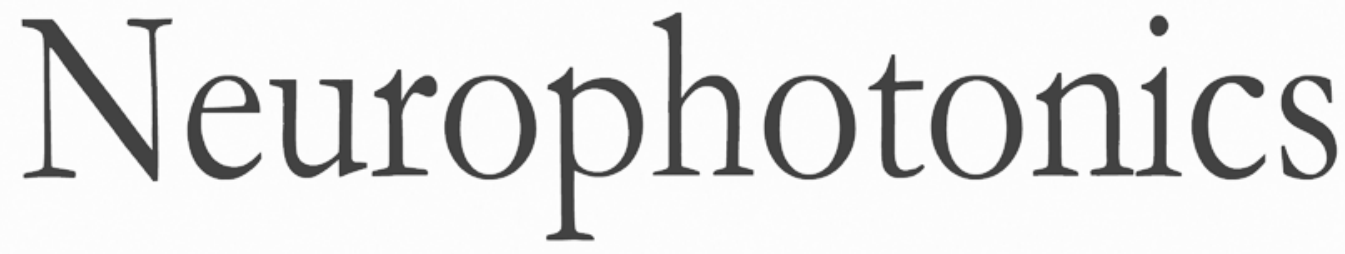

\title{
Somatic stimulation causes frontoparietal cortical changes in neonates: a functional near-infrared spectroscopy study
}

Nasser H. Kashou

Irfaan A. Dar

Kathryn A. Hasenstab

Ramzi W. Nahhas

Sudarshan R. Jadcherla 


\title{
Somatic stimulation causes frontoparietal cortical changes in neonates: a functional near-infrared spectroscopy study
}

\author{
Nasser H. Kashou, ${ }^{a, \star}$ Irfaan A. Dar, ${ }^{a, b}$ Kathryn A. Hasenstab, ${ }^{b}$ Ramzi W. Nahhas, ${ }^{\text {c,d }}$ and Sudarshan R. Jadcherla ${ }^{b, e}$ \\ ${ }^{a}$ Wright State University, Biomedical, Industrial and Human Factors Engineering, 3640 Colonel Glenn Highway, Dayton, Ohio 45435, United States \\ ${ }^{\text {b}}$ The Research Institute at Nationwide Children's Hospital, Innovative Research Program in Neonatal and Infant Feeding Disorders, 700 Children's \\ Drive, Columbus, Ohio 43205, United States \\ 'Wright State University, Department of Community Health, 3123 Research Boulevard, Kettering, Ohio 45420, United States \\ dWright State University, Department of Psychiatry, 3123 Research Boulevard, Kettering, Ohio 45420, United States \\ ${ }^{\mathrm{e} T}$ The Ohio State University, College of Medicine, Division of Neonatology, Department of Pediatrics, 370 West 9th Avenue, Columbus, Ohio 43210, \\ United States
}

\begin{abstract}
Palmar and plantar grasp are the foremost primitive neonatal reflexes and functions. Persistence of these reflexes in infancy is a sign of evolving cerebral palsy. Our aims were to establish measurement feasibility in a clinical setting and to characterize changes in oxyhemoglobin $(\mathrm{HbO})$ and deoxyhemoglobin $(\mathrm{HbD})$ concentration in the bilateral frontoparietal cortex in unsedated neonates at the crib-side using functional near-infrared spectroscopy (fNIRS). We hypothesized that bilateral concentration changes will occur upon somatic central and peripheral somatic stimulation. Thirteen preterm neonates (five males) underwent time 1, and six (two males) returned for time 2 (mean PMA $=41.6$ and 47.0 weeks, respectively). Signals from a total of 162 somatic stimuli responses were measured. Response amplitude, duration, and latency were log-transformed and compared between palmar, plantar, and oromotor stimuli using linear mixed models, adjusted for cap, electroencephalogram abnormality, time (1 versus 2), and Sarnat score, if necessary. The oromotor stimulus resulted in a $50 \%$ greater response than the palmar or plantar stimuli for $\mathrm{HbO}$ left and right hemisphere duration $(p<0.0001)$. There were no other statistically significant differences between stimuli for any other outcome $(p>0.05)$. Utilizing fNIRS in conjunction with occupational and physical therapy maneuvers is efficacious to study modifiable and restorative neurophysiological mechanisms. ๑ 2016 Society of Photo-Optical Instrumentation Engineers (SPIE) [DOI: 10 .1117/1.NPh.4.1.011004]
\end{abstract}

Keywords: functional near-infrared spectroscopy; neonates; activation; somatic stimulation.

Paper 16012SSR received Mar. 1, 2016; accepted for publication May 2, 2016; published online Aug. 17, 2016.

\section{Background}

Prematurity adversely affects the developing neonatal brain and is manifested by guarded neurodevelopmental outcomes ${ }^{1-3}$ and behavioral and cognitive deficits. ${ }^{4-6}$ Occupational therapy in these high-risk infants has been shown to improve the rate of development in somatic tasks, such as sucking. ${ }^{7}$ However, the acute effects of occupational therapies are not quantifiable, and the effects of such therapies on the cerebral cortex are not clear. Thus, functional measurements are required to enhance our understanding of the sensory-motor effects of peripheral somatic stimulation and to define cortical involvement. Functional near-infrared spectroscopy (fNIRS) is a modality that permits the study of functional brain responses in neonates. Unlike magnetic resonance imaging (MRI), NIRS was recognized to be useful at the bedside ${ }^{8-10}$ to monitor changes in cerebral perfusion and oxygenation, particularly in sick infants who have dynamic pathophysiology of the brain. ${ }^{11,12}$ Neonatal occupational and physical therapies have evolved over time with emphasis on sensory-motor integration and improvement in motor functions. In the convalescing neonatal intensive care unit (NICU) infant, commonly used approaches are passive

*Address all correspondence to: Nasser H. Kashou, E-mail: Nasser. Kashou@ wright.edu touch, containment (bundling in a blanket), and stimulation of the palmar and plantar aspects of the palms and feet. The effects of therapies on developmental changes in the brain are not known, although the effects of somatic stimulation with passive touch studies using functional magnetic resonance imaging (fMRI) have been studied. ${ }^{13,14}$ However, fMRI studies are not commonly feasible in NICU neonates, and it is difficult to control the implementation of experimental paradigms. This is largely due to the expense, need for transport, need for personnel with technical skills, movement artifacts, need for longitudinal studies, and analytical difficulties owing to rapid changes during maturation and growth. On the other hand, implementing and repeating these studies using fNIRS can prove advantageous, as these methods offer the ability to monitor localized cerebral cortical activation by measuring changes in the concentration of oxyhemoglobin $(\mathrm{HbO})$ and deoxyhemoglobin $(\mathrm{HbD})$ in a portable, noninvasive manner at the cribside regardless of the activity state and nature of pathophysiology. These approaches using fNIRS in conjunction with occupational and physical therapy maneuvers may be efficacious to study the modifiable and restorative neurophysiological mechanisms. The ability to monitor the brain noninvasively has made NIRS favorable for measuring cerebral oxygenation, quantifying cerebral

$2329-423 X / 2016 / \$ 25.00$ (C) 2016 SPIE 
hemodynamics, i.e., cerebral blood flow and cerebral blood volume ${ }^{15}$ and measuring other circulatory parameters. ${ }^{16}$ Therefore, this study was undertaken specifically to elucidate functional developmental processes in the newborn brain with reference to the effects of somatic stimulation and to assess the feasibility of NIRS in the clinical NICU setting.

\subsection{Significance}

Functions of the frontoparietal cerebral cortex involve multiple interactions in producing a final behavior, and such interactions involve afferent and efferent neural circuitry in addition to perfusion to the regions of interest. Importantly, cortical perception is fundamental to the development of cognition, ${ }^{17}$ memory, ${ }^{18}$ defense reflexes, ${ }^{19}$ integration, and execution of final actions that culminate in sensory-motor patterns manifesting as and in addition to physiological and behavioral responses. ${ }^{20}$ Representation of palms, feet, and tongue is wider within the frontoparietal cortex than in the vicinity of the precentral and postcentral gyrus. Although the importance of the mentioned functions from adult human brain studies is increasingly understood using functional MRI studies, there remains a gap in the understanding of such functions in the human neonate. This gap in knowledge is largely due to the difficulties in keeping neonates still in an unsedated state and the need to attend to these neonates promptly in order to maintain cardiorespiratory stability. Recently, fNIRS methods have been applied to study cerebral cortical functions across the age spectrum. ${ }^{21-24}$ Thus, in the current study, we characterized the physiological changes in the neonatal brain following each of three somatosensory stimulation methods: plantar (foot tapping), palmar (hand tapping), and oromotor (pacifier).

Our main objective was to acquire NIRS in unsedated neonates at the bedside and analyze changes in $\mathrm{HbO}$ and $\mathrm{HbD}$ concentrations in response to the applied somatic stimuli. Because the bilateral frontoparietal region of interest is the main sensorymotor association area, we hypothesized that significant $\mathrm{HbO}$ and $\mathrm{HbD}$ concentration changes will occur in the regions of interest within the brain associated with specific targeted somatic stimulation. Additionally, we compared the effects of plantar, palmar, and oromotor stimulation.

\section{Methods}

\subsection{Participants}

Participants were 13 neonates (five males) born at $39.3 \pm 0.6$ (mean $\pm \mathrm{SE}$ ) weeks gestation age (GA) with mean postmenstrual age (PMA) of $41.6 \pm 0.7$ (mean $\pm \mathrm{SE}$ ) weeks at time 1 studies. Six (two males) at mean PMA of $47.0 \pm 1.1$ (mean $\pm \mathrm{SE}$ ) weeks came back for time 2 studies. All procedures and protocols were approved by the Institutional Research Review Board at Nationwide Children's Hospital Research Institute, Columbus, Ohio. Written informed consent and HIPAA authorization were obtained from the parents prior to the study. The 13 neonates had birth weight $3.4 \pm 1.9 \mathrm{~kg}$. The frontooccipital circumference (FOC) at times 1 and 2 evaluations was $34.5 \pm 0.5 \mathrm{~cm}$ and $36.6 \pm 0.8 \mathrm{~cm}$, respectively (Table 1). Continuous data were collected while neonates went through variable intervals of sleep and wake states with periods of applied stimuli with a total of 162 somatic stimuli response measurements.

Table 1 Patient demographics. GA, gestational age in weeks; FOC, frontooccipital circumference in centimeters; and PMA, postmenstrual age in weeks. T1, time 1 and T2, time 2. Patient 3 did not show any response.

\begin{tabular}{|c|c|c|c|c|c|c|c|c|c|}
\hline Patient & Gender & $\begin{array}{c}\text { GA } \\
\text { (wks) }\end{array}$ & $\begin{array}{l}\text { Birth } \\
\text { weight } \\
\text { (g) }\end{array}$ & $\begin{array}{l}\text { Sarnat } \\
\text { score }\end{array}$ & $\begin{array}{c}\text { electroencephalogram } \\
\text { (EEG) } \\
\text { abnormality }\end{array}$ & $\begin{array}{l}\text { FOC } \\
@ \text { T1 } \\
(\mathrm{cm})\end{array}$ & $\begin{array}{l}\text { PMA } \\
\text { @ T1 } \\
\text { (wks) }\end{array}$ & $\begin{array}{l}\text { FOC } \\
@ \text { T2 } \\
(\mathrm{cm})\end{array}$ & $\begin{array}{l}\text { PMA } \\
\text { @ T2 } \\
\text { (wks) }\end{array}$ \\
\hline 1 & Boy & 35.0 & 2101 & III & $\mathrm{Y}$ & 30.5 & 37.9 & na & na \\
\hline 2 & Girl & 37.0 & 2225 & II & $\mathrm{Y}$ & 33 & 39.1 & na & na \\
\hline 3 & Girl & 40.9 & 4601 & III & $\mathrm{Y}$ & 35.6 & 44.4 & na & na \\
\hline 4 & Boy & 39.0 & 3700 & II & $\mathrm{Y}$ & 36 & 40.0 & na & na \\
\hline 5 & Girl & 37.9 & 2764 & I & $\mathrm{Y}$ & 32.5 & 40.0 & 34.6 & 44.0 \\
\hline 6 & Girl & 39.3 & 3532 & II & $\mathrm{Y}$ & 35.7 & 39.0 & 36 & 45.0 \\
\hline 7 & Boy & 38.0 & 3501 & II & $\mathrm{N}$ & 35.5 & 42.0 & na & na \\
\hline 8 & Boy & 40.9 & 3270 & II & $\mathrm{Y}$ & 38.2 & 46.0 & - & 50.0 \\
\hline 9 & Girl & 41.3 & 3650 & II & $\mathrm{Y}$ & 35.5 & 43.0 & na & na \\
\hline 10 & Boy & 41.0 & 3855 & II & $\mathrm{N}$ & 33 & 42.0 & 38.8 & 46.4 \\
\hline 11 & Girl & 41.0 & 3500 & II & $\mathrm{Y}$ & 35 & 41.9 & na & na \\
\hline 12 & Girl & 41.7 & 3420 & II & $\mathrm{Y}$ & 35 & 44.9 & 37 & 50.7 \\
\hline 13 & Girl & 38.4 & 3570 & III & $Y$ & 33.5 & 40.4 & 36.5 & 45.6 \\
\hline Mean & & 39.3 & 3361 & 2.2 & & 34.5 & 41.6 & 36.6 & 47.0 \\
\hline
\end{tabular}




\subsection{Study Methods}

A continuous wave single-phase compact NIRScout imaging system (NIRx Medical Technologies LLC)—with four 760 and $850 \mathrm{~nm}$ wavelength LED sources (power: $>5 \mathrm{~mW} /$ wavelength) and eight $\mathrm{Si}$ photodiode detectors (sensitivity and dynamic range: $<1 \mathrm{pW}, 90 \mathrm{~dB}$ )—was used to measure changes in $\mathrm{HbO}$ and $\mathrm{HbD}$. A noninvasive NIRS-EEG cap encompassing the sources and detectors was placed onto the surface of the neonate's head by the neonatologist and comfortably secured via elastic straps. The source-detector (channel) layout covered the frontoparietal cortical regions [Fig. 1(a)]. The cap size varied based on the neonate's head circumference.

\subsection{Experimental Protocol}

Based on the infant's head circumference at evaluation, the corresponding cap was fitted with the optical sources and detectors. A neonatologist placed and adjusted the cap to ensure proper positioning. Contact between the scalp and optodes was assessed by calibrating the gain setting on the NIRx system and physically adjusted, if needed. All studies were performed in a neonatal nursery setting; as per NICU environment protocols, the ambient temperature and light intensity were fairly regulated during the study. The sources and detectors were placed on the frontoparietal regions of the brain as determined by the surface markings and discussion with neonatologist and pediatric neuroradiologist and also further clarified based on an MRI atlas ${ }^{25}$ and correlations between adult and infant neuroanatomy. ${ }^{26}$ True sleep states were not discerned since concurrent polysomnography was not done.

The overall experimental paradigm included targeted palmar (hand) and plantar (foot) stimulation applied to the left and right sides using finger and foot tap [Fig. 1(b)]. The neonatologist performed these somatic stimuli, tapping with a frequency of about $1 \mathrm{~Hz}$. At least $30 \mathrm{~s}$ of baseline was obtained before the stimulus was given. The stimulus duration was varied from 10 to $30 \mathrm{~s}$ (mean of $15 \mathrm{~s}$ ) with a minimum of $30 \mathrm{~s}$ rest. In addition, oromotor stimulation (pacifier) was provided at random intervals to soothe the subject with at least $30 \mathrm{~s}$ of rest. Bilateral changes in $\mathrm{HbO} / \mathrm{HbD}$ concentrations from baseline values in the frontoparietal cortical region were analyzed. This included response effects of 162 (66 palmar, 75 plantar, and 21 oromotor) somatic stimuli measured from all patients.

(a)

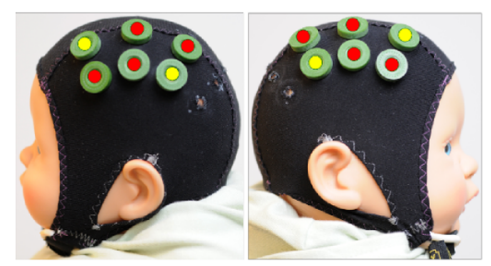

(b)

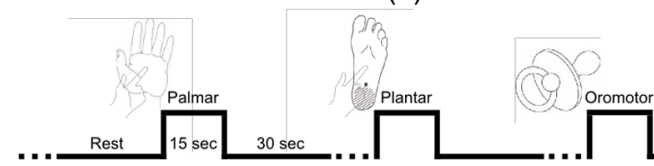

Fig. 1 (a) Representation of source-detector (channel) layout utilized to cover frontoparietal cortex. Yellow indicates sources and red indicates detectors. (b) The study design included oromotor (pacifier), palmar, and plantar tapping for $15 \mathrm{~s}$ of stimulation and $30 \mathrm{~s}$ of rest interspersed randomly. (c) HDR definitions used in calculations.

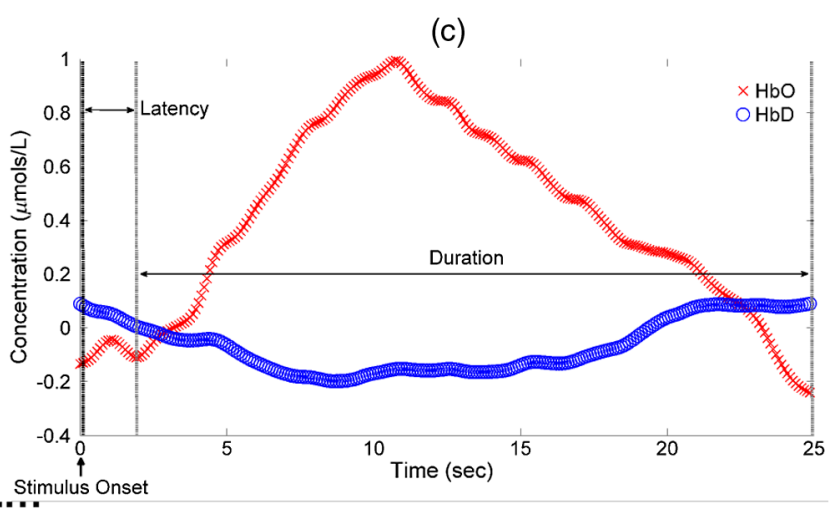

\subsection{Near-Infrared Spectroscopy Data Analysis}

$\mathrm{HbO}$ and $\mathrm{HbD}$ concentration $(\mu \mathrm{mol} / L)$ changes were determined and analyzed for potential trends [Fig. 1(c)]. The following characteristics were defined to evaluate the feasibility of NIRS: "Baseline" was defined as the rest period when the infant was noted to be quiet and still before a stimulus was given. A baseline value was obtained immediately before the onset of the stimulus. "Response latency time" was defined as the time from the onset of the stimulus to the beginning of the stimulusinduced response. "Hemodynamic response (HDR)" was defined as an increase in the $\mathrm{HbO}$ concentration level and a decrease in the $\mathrm{HbD}$ concentration level in response to a given stimulus. "Response duration" was defined as the time from the onset of the stimulus-induced response to the time the $\mathrm{HbO} / \mathrm{HbD}$ concentrations returned to baseline normal levels. Response duration and latency between each type of stimulus were analyzed. Postprocessing was done using Homer2 and code written in MATLAB (The Mathworks, Inc.). ${ }^{27}$

A three-step process was used to remove motion artifacts, both quantitatively and qualitatively, due to the erratic nature of the infants during the studies. Motion artifacts were determined both during the study and the signal analysis. Undesired events such as crying or unwanted motion were marked down during the study, and trials that contained these issues were discarded and acquired again when the infant was in a calm state. Motion artifacts were removed with the wavelet filter using an interquartile range value of 0.1 , after converting the data to optical density. ${ }^{28}$ This was followed by a bandpass filter from 0.02 to $0.75 \mathrm{~Hz}$, and then the signal was converted to hemoglobin concentration based on the modified Beer-Lambert law (see Appendix). Finally, during signal analysis of HDRs, any stimuli that showed motion artifacts (spike noise or slow-moving drift of $\mathrm{HbO}$ and $\mathrm{HbD}$ ) were removed from the analysis.

Signals that were not skewed with motion artifacts and showed HDR were selected from the data based on Homer2 and visual analysis. These signals were averaged across all patients and zeroed by subtracting the mean of the values of $t<0$. The duration analyzed of each response was confined to $45 \mathrm{~s}$ for every stimulus type. HDRs were then determined by visually inspecting the averaged signals. The difference between baseline and maximum value, latency, and response duration was marked down for each channel that showed a response. 


\subsection{Statistical Analysis}

The outcome variables were $\mathrm{HbO}$ and $\mathrm{HbD}$ concentration changes (amplitude) between the baseline and the stimulusinduced response, response duration, and response latency. These outcomes were compared by response side (left or right) between the three stimulus types: plantar, palmar, and oromotor. Comparisons were made using a linear mixed regression model with a random infant effect to account for the serial correlation due to repeated measures on the same individuals. Due to non-normality of residuals, outcome variables were log-transformed prior to testing for significant differences. This transformation is possible only on positive outcomes; the latency outcome included some zeros, so 1 was added prior to transformation; amplitude for $\mathrm{HbD}$ included some negative values and was on a very small scale, so the minimum value +0.000001 was added prior to transformation.

We adjusted for cap (1 versus 2 versus 3), EEG abnormality (yes/no), time (1 versus 2), and Sarnat score (I versus II versus III) by including them in the model if their inclusion resulted in a decrease in Akaike's Information Criteria. ${ }^{29}$

The unadjusted regression model was

$$
\begin{aligned}
Y_{i j}= & \beta_{0}+\beta_{1} I\left(\text { Stimulus }_{i j}=\text { Palmar }\right) \\
& +\beta_{2} I\left(\text { Stimulus }_{i j}=\text { Plantar }\right)+b_{i}+\varepsilon_{i j},
\end{aligned}
$$

where $Y_{i j}$ is the transformed outcome for the $j$ 'th response for individual $i, \beta_{0}$ is the mean outcome for the oromotor stimulus, $\beta_{1}$ is the difference in mean response between the palmar and oromotor stimuli, $\beta_{2}$ is the difference in mean response between the plantar and oromotor stimuli, the difference in mean outcome between palmar and plantar stimuli is represented as $\beta_{1}-\beta_{2}, I$ (condition) is an indicator function that takes the value 1 if the condition is true and 0 otherwise, $b_{i}$ (normally distributed with mean 0 ) is the random infant effect representing heterogeneity between infants and the inclusion of which accounts for the nonindependent nature of the data (for a given outcome, repeated measures on the same individual are correlated), and $\epsilon_{i j}$ (normally distributed with mean zero) is the error term. Adjusted models included terms for the variable being adjusted for.

Three extreme outliers were excluded from the analysis: a value of $2.64 \times 10^{-5}$ for $\mathrm{HbO}$ amplitude on the left response side (the next largest value was $3.85 \times 10^{-6}$ ), a value of $-1.13 \times$ $10^{-5}$ for $\mathrm{HbD}$ amplitude on the left response side (the next most negative value was $-2.22 \times 10^{-6}$ ), and a value of $-3.17 \times 10^{-6}$ for $\mathrm{HbD}$ amplitude on the right response side (the next most negative value was $-1.67 \times 10^{-6}$. Removal of these outliers did not impact our conclusions regarding the comparison of outcomes between stimuli.

Significance of differences between stimuli was tested at the $\alpha=0.05$ level of significance. $\mathrm{R}$ version $3.1 .2^{30}$ was used for statistical analysis.

\section{Results}

Of the 162 stimuli, 95 (37 palmar, 42 plantar, and 16 oromotor) evoked responses in either left, right, or both hemispheres, and one of the 13 patients did not show any responses. HDR amplitude, latency, and duration for $\mathrm{HbO}$ following stimuli, including the number of responses per hemisphere, are summarized in Table 2. The HDR amplitude for HbD is also tabulated. The typical HDRs (mean $\pm \mathrm{SE}$ ) seen for palmar, plantar, and oromotor stimuli are shown in Fig. 2.

There were significant differences between time 1 and time 2 for $\mathrm{HbO}$ latency (left hemisphere), between those with and without an EEG abnormality for $\mathrm{HbO}$ response amplitude (right hemisphere), and between caps for $\mathrm{HbO}$ duration (right hemisphere). However, the significance of differences between stimuli was not affected by adjustment for any covariate.

No significant differences in response amplitude of $\mathrm{HbD}$ and $\mathrm{HbO}$ across all three stimuli, or between left and right hemispheres, were seen (Fig. 3). No significant differences in

Table 2 Summary table of mean HDR amplitude, latency, and duration as a result of plantar, palmar, and oromotor stimuli in the left and right hemisphere. No hemispheric differences were seen within stimuli. Oromotor response duration was significantly larger than that of plantar or palmar.

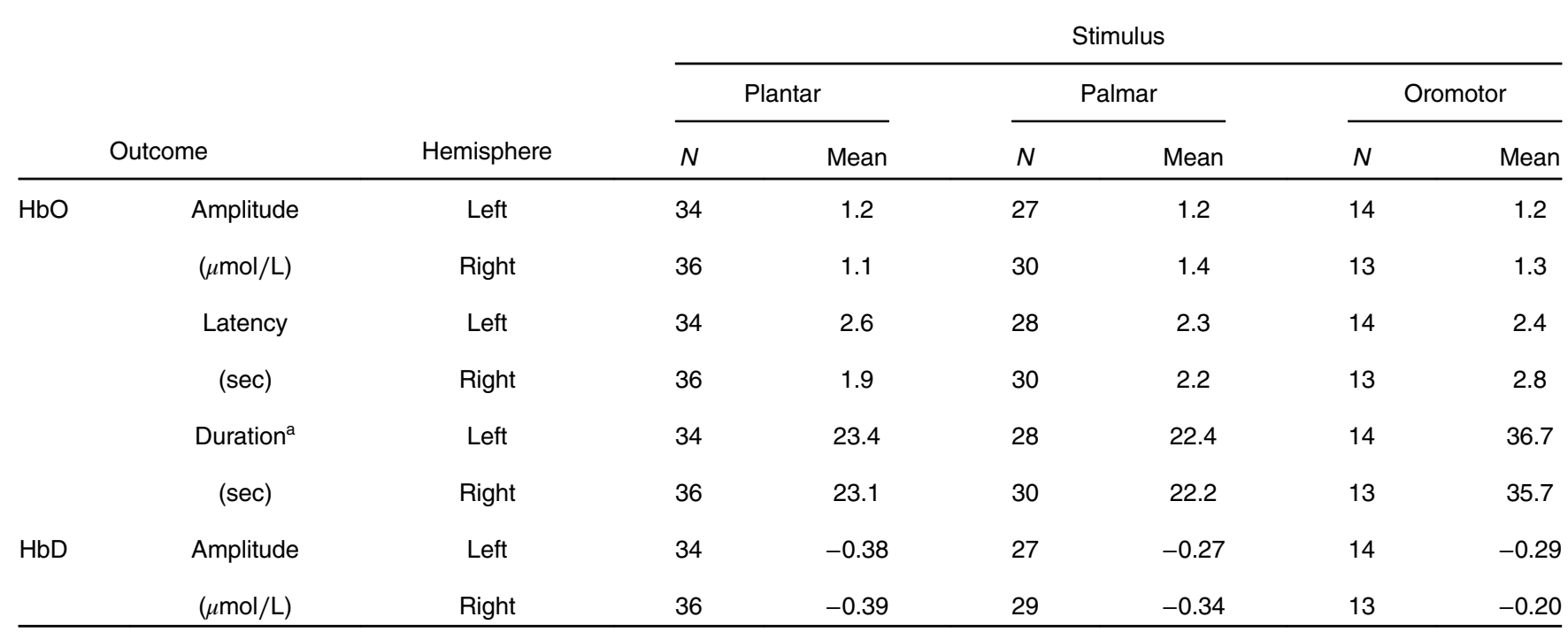

${ }^{a}$ For duration, the response following the oromotor stimulus was significantly greater than the responses following plantar $(p<0.0001)$ and palmar $(p<0.0001)$ for each response side. 

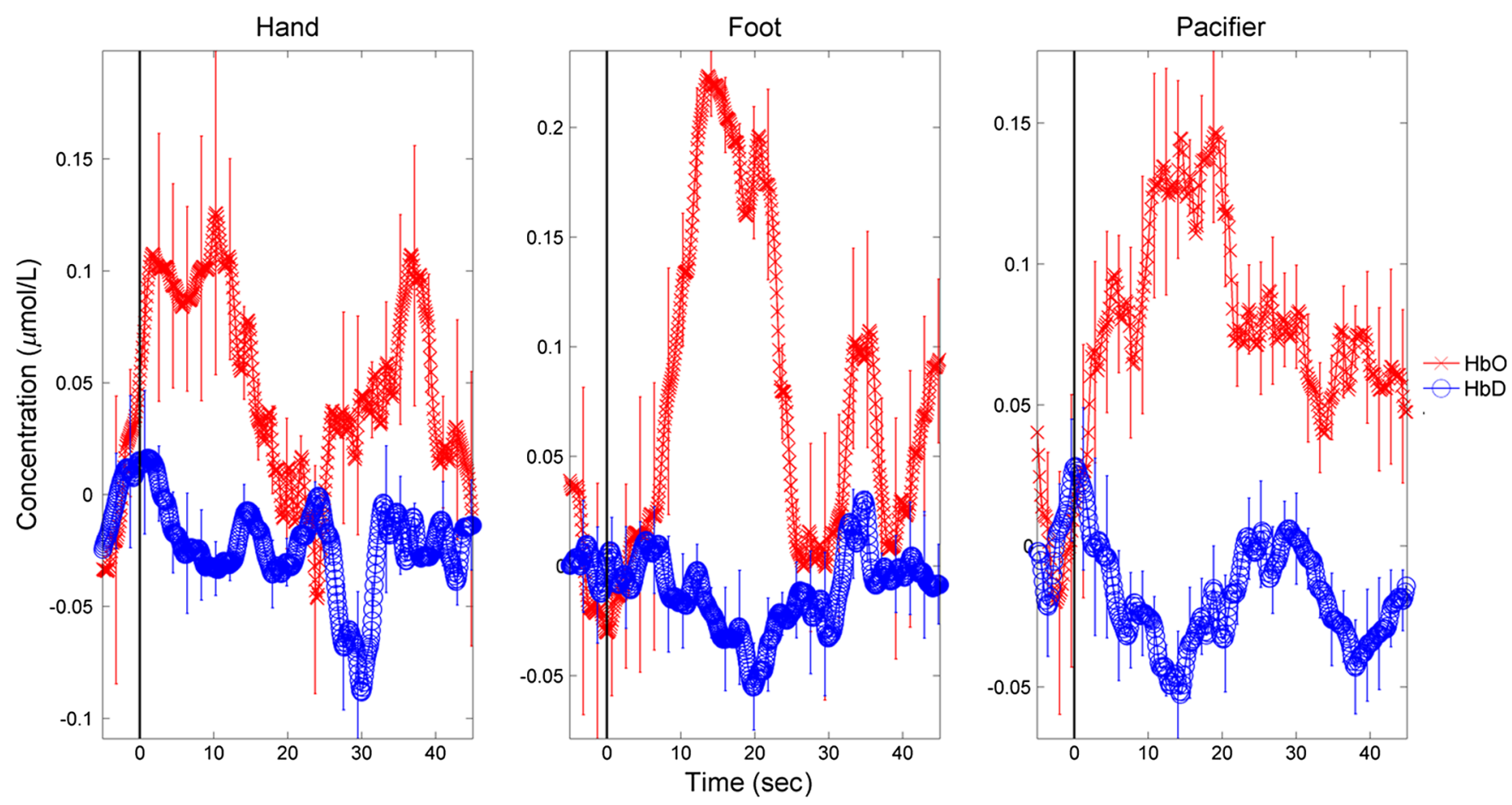

Fig. 2 The typical HDRs (mean \pm SE) seen for palmar, plantar, and oromotor stimuli (HbO-red, $\mathrm{HbD}-$ blue). The $y$-axis indicates the concentration changes in $\mu \mathrm{mol} / \mathrm{L}$.

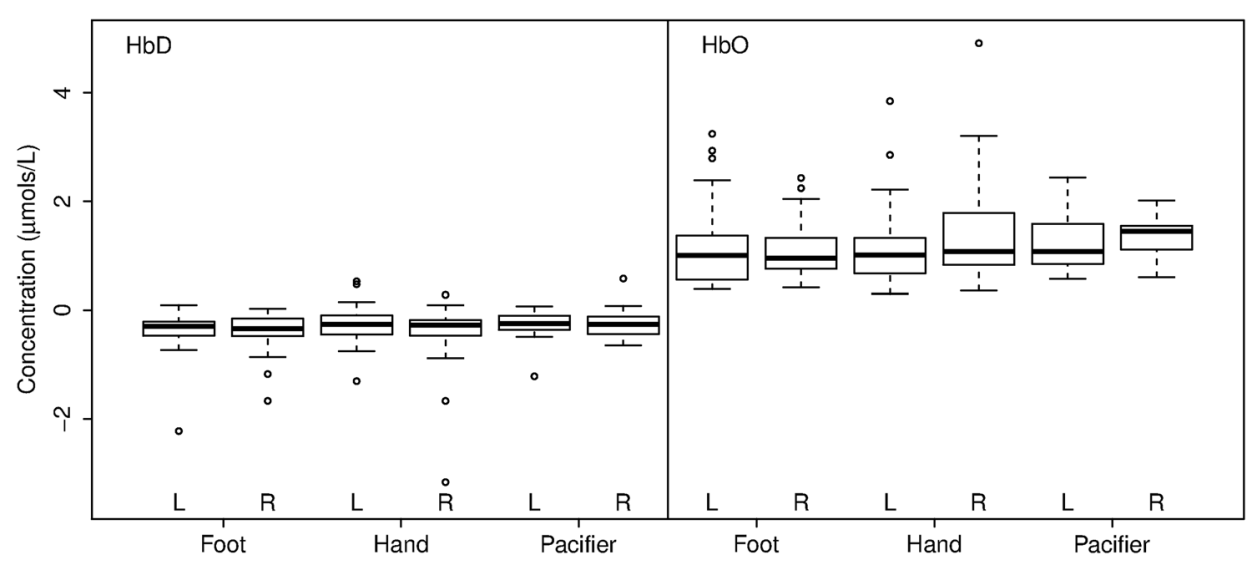

Fig. $3 \mathrm{HbD}$ and $\mathrm{HbO}$ response amplitude ( $\mu \mathrm{mol} / \mathrm{L}$ ) for palmar, plantar, and oromotor stimuli. No significant differences across all three stimuli and between left and right hemispheres were seen.

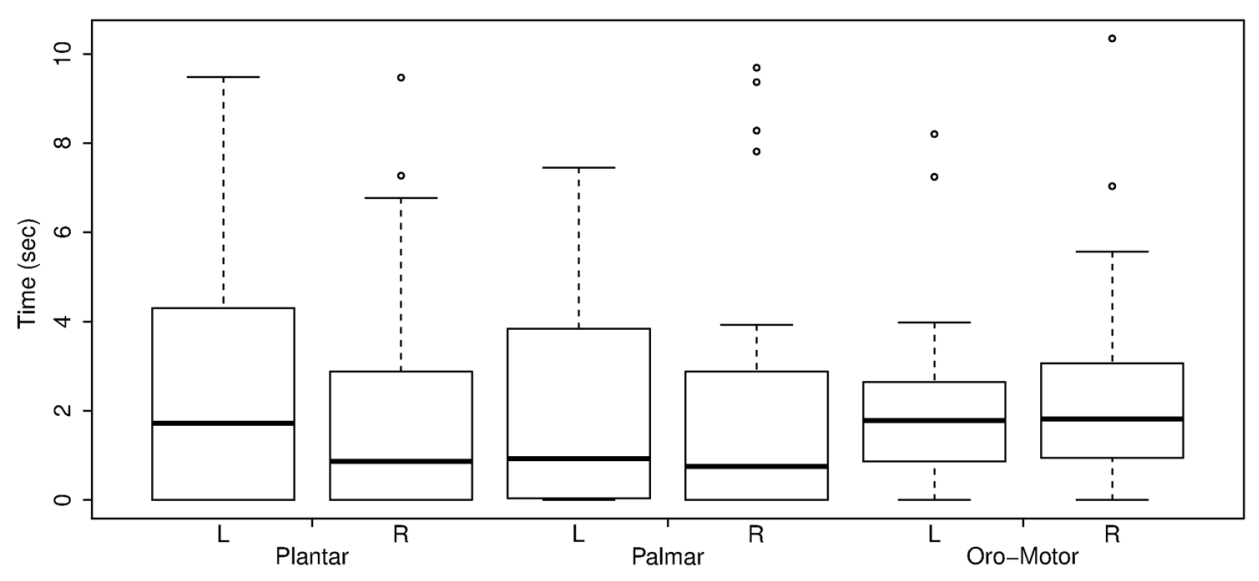

Fig. $4 \mathrm{HbO}$ response latency (sec) for palmar, plantar, and oromotor stimuli. No significant differences in response latency across all three stimuli and between left and right hemispheres were seen. 


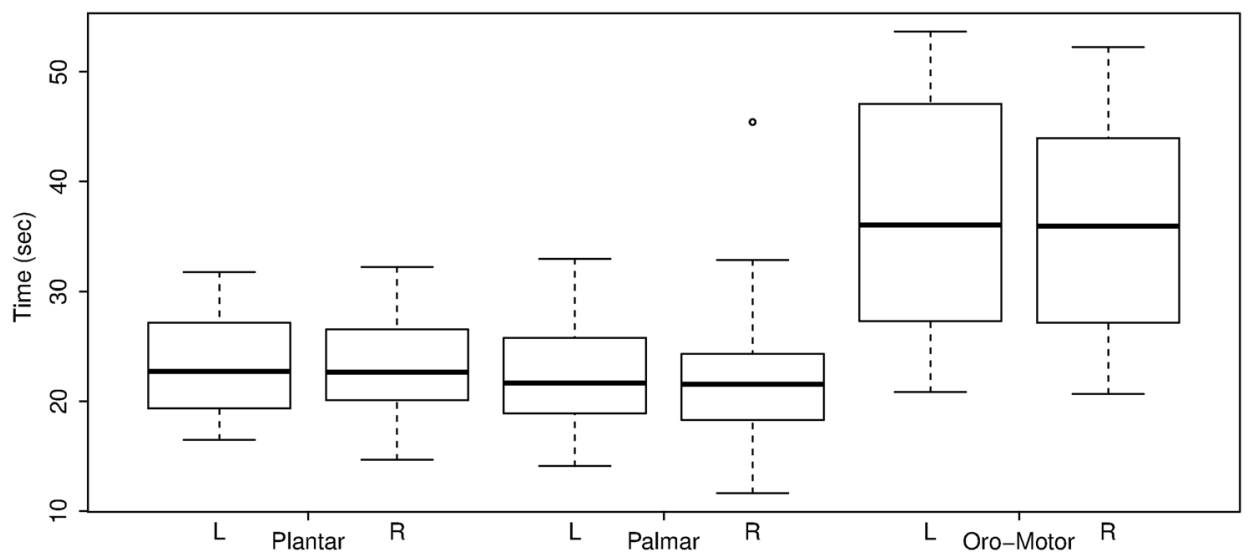

Fig. $5 \mathrm{HbO}$ response duration (sec) for palmar, plantar, and oromotor stimuli. The oromotor stimulus resulted in a $50 \%$ greater response than the palmar or plantar stimuli (adjusted for cap) for $\mathrm{HbO}$ left and right hemisphere duration $p<0.0001$. No significant differences in response latency between left and right hemispheres were seen.

response latency across all three stimuli, or between left and right hemispheres, were seen (Fig. 4). No significant differences in response duration between left and right hemispheres were seen (Fig. 5). However, the oromotor stimulus resulted in a significantly greater response than the palmar or plantar tapping stimulus (adjusted for cap) left hemisphere duration $[\exp (0.47)=1.60 \times$ greater than palmar, $p<0.0001 ; \exp (0.43)=$ $1.54 \times$ greater than plantar, $p<0.0001]$, and right hemisphere duration $[\exp (0.44)=1.55 \times$ greater than palmar, $p<0.0001$; $\exp (0.39)=1.48 \times$ greater than plantar, $p<0.0001]$ (Fig. 5).

\section{Discussion}

The salient findings of our study are: (1) as hypothesized, bilateral responses were present and no significant hemispheric differences in amplitude, latency, or duration were seen for any stimulus, and (2) analysis of the response duration demonstrated significant differences between the oromotor stimulus and each of the palmar and plantar stimuli.

Tactile stimulation of infants has been shown to reduce length of stay. ${ }^{31}$ By understanding the pathways and cortical activation of these neonates during focused stimulation, targeted focal areas can be determined that correlate to high activation and help to reduce length of stay. Differences between efficient and inefficient feeders have been illustrated for both nutritive and non-nutritive sucking. ${ }^{32}$ Non-nutritive sucking and tactile stimulation have also been shown to reduce length of stay and improve neonates' oral feeds. ${ }^{31,33,34}$ NIRS can help elucidate the cortical readings and focal areas where these treatments work. The neural pathway and coordination in regards to feeding are not fully understood in neonates, so more functional research is required. It was observed that both tactile and oromotor stimulation of certain regions has improved oral feeds compared to no stimulation, ${ }^{34}$ but underlying cortical changes were not obtained. Understanding the synaptic genesis at these targeted cortical areas when provided with such stimulation can help to uncover which treatments are most beneficial.

We have demonstrated the feasibility of using NIRS in the neonatal setting; however, we recognize there is room for further advances. For instance, some signals had to be discarded because of motion artifacts. Hence, additional optimization is possible from both the hardware and software perspectives. Understanding what has worked in the past and what has not is a crucial step in moving forward. Additional concerns with
NIRS are the signal-to-noise ratio (SNR) and signal quality, and several studies ${ }^{35}$ approach these challenges differently.

There has been a variety of investigations on the use of NIRS on neonates in both functional and clinical studies to determine brain activation or cerebral oxygen consumption. To our knowledge, ours is the first to investigate and compare responses to palmar, plantar, and oromotor stimuli using NIRS. Numerous NIRS studies deal with visual or voice recognition and how the infant's brain interprets these stimuli. ${ }^{36-43}$ Other researchers have used NIRS in conjunction with other data collection instruments in order to help characterize tissue oxygen saturation of neonates with disorders. ${ }^{44-46}$ Also, NIRS can be applied to therapeutic neuromotor assessment techniques. ${ }^{47}$

Importantly, no sedation was used, which is a common practice during MRI infant studies. ${ }^{38}$ This allowed us to use stimulations to determine cortical activation. ${ }^{36}$ NIRS can also be used in conjunction with other data acquisition tools, which can help validate and give more insight into the received signals. ${ }^{44,48,49}$

\subsection{Implications}

Near-infrared spectroscopy is a promising field for bedside neonatal monitoring. Cortical changes in regions of specificity and neural development can be analyzed. Understanding how the maturation of the neonatal brain affects neural pathways can clarify the usage of therapeutic techniques that promote cortical activation.

1. Tactile stimulation has been used to provide physical and occupational therapy in the clinical setting, but understanding which brain areas are involved and the reasons behind it are not fully understood.

2. Oral feeding plays an important role in infants' feeding abilities; thus, development of suck-swallow rhythms is crucial. Non-nutritive sucking using pacifiers has improved oral feeds and helped reduce length of stay. ${ }^{33,34}$ How non-nutritive sucking contributes to the neural pathway of feeding is not as fully understood as is the swallow pathway in neonates. Concurrent NIRS during oral stimulation can help elucidate these issues. 
3. While, in this study, NIRS was used to analyze somatic stimulation, it can be also be used in other ways. By showing that results were obtained with NIRS in the NICU setting, future studies that address functional activity can benefit from the portability and ease of use of this imaging modality in this setting.

\subsection{Limitations and Future Directions}

When studying high-risk infants, safety considerations are essential. In addition to the comprehensive knowledge requirements of the NICU by the occupational therapist, ${ }^{50}$ training on and understanding the NIRS setup would need to be considered. Technical difficulties with NIRS appeared during the studies. Motion artifacts, a problem when imaging infants, ${ }^{51}$ may bias the results by masking the true changes in $\mathrm{HbO}$ and $\mathrm{HbD}$. These artifacts also affected the baseline data in some trials and, in certain cases, caused the cap to loosen, which reduced the total time for data acquisition. The motion artifacts that remained in the usable data sets were mostly alleviated by the wavelet and bandpass filter, but stimuli that still contained motion artifacts were removed from the analysis. Research protocols need to be designed to keep the infant happy and calm in order to reduce any unwanted motion or behavior that may arise if the infant becomes agitated. ${ }^{51}$ Initially, some of our artifacts were not specifically from the infant moving, rather from the bed being disturbed inadvertently by the clinical staff. In this case, simply informing them to be more cognizant of the sensitivity of the system would improve data collection and use. In addition, hair obstruction lowers the SNR because the source light intensity is lowered by interactions with the hair, ${ }^{40,51}$ but is not a serious problem with neonates due to the sparse amount of fine hair. Another major problem with NIRS research is that there is no standard for baseline values of $\mathrm{HbO}$ and $\mathrm{HbD},{ }^{46,52}$ which leads to a lack of reproducibility in research. ${ }^{17}$ This is due, in part, to different hardware among researchers. ${ }^{52}$ It is also due to the fact that every infant brain is different because this human period is one of development, so there is no standard model of an infant's brain. ${ }^{51}$ The variability in these studies highlights this reproducibility problem since there are different hardware systems and wavelengths used to measure $\mathrm{HbO}$ and $\mathrm{HbD}$. Furthermore, infants in different studies were of different ages, which further causes variations since the neuroanatomy is quickly developing as opposed to a mature adult brain. The differences between controls and patients are not known at this point in time, and will need to be investigated. Future studies must consider the tradeoffs between number of channels, patient comfort, and spatial and time resolution. Also, larger sample sizes with more repeated measurements will clarify intra- and intersubject variability.

\section{Conclusion}

The use of fNIRS at the bedside in unsedated neonates can prove to be advantageous for studying newborn brain development. Specifically, this modality can be used to assess the effectiveness of certain therapies on the neurophysiological level and allow a new perspective and methodology for future treatment. In this study, no significant hemispheric difference was seen regarding response amplitude, duration, and latency within stimuli. However, response duration was greater for pacifier than foot or hand tapping. To the best of our knowledge, this is the first study to investigate palmar, plantar, and oromotor stimuli on infants using NIRS.

Our study may assist with cribside monitoring and evaluation of practices. Specifically, the NIRS modality can be utilized to assess the effectiveness of certain therapies on the neurophysiological connectivity and integration of organ functions. For example, integrating fNIRS with time-synchronized somatic stimuli may permit assessment of occupational and physical therapies on the infant's regional brain activity, functional connectivity changes, muscle memory, sensitivity, responsiveness, and magnitude of responses. These assessment methods may also be useful to determine the effects of maturation and neuromotor malfunction. Future studies utilizing concurrent swallowintegrated EMG and pharyngo-esophageal manometry with fNIRS may allow for brain connectivity mapping related to normal infant feeding and dysphagia-related conditions.

Particularly, we will be able to assess the duration and magnitude of brain response during solitary and repetitive stimuli. Prolonged activation implies prolonged neurophysiologic and HDR on fNIRS. In those with neonatal stroke, it is important to restore motor functions of skeletal muscles involved with specific tasks. The larger question is to test how different therapies generate changes with acute and chronic functional connectivity. This may form the basis of measuring neuroplasticity.

\section{Appendix}

To determine $\mathrm{HbO}$ and $\mathrm{HbD}$ concentrations, the modified BeerLambert law is used. ${ }^{53}$

$I=I_{0} e^{-\varepsilon[C] L}$,

$\Delta \mathrm{OD}=-\ln \left(I_{\text {final }} / I_{\text {initial }}\right)=\varepsilon \Delta \mathrm{CLB}$,

where $I$ is the detected light intensity, $I_{0}$ is the incident intensity, $\varepsilon$ is the extinction coefficient, $C$ is the concentration of the chromophore, $L$ is the optical path length, OD is the optical density, and $B$ is the differential path-length factor.

This equation is further modified to give the change in concentration for $\mathrm{HbO}$ and $\mathrm{HbD}$ as

$$
\begin{aligned}
\Delta[\mathrm{HbD}]= & {\left[\left\{\left[\varepsilon_{\mathrm{HbO}}^{\lambda 2}\left(\Delta O D^{\lambda 1} / B^{\lambda 1}\right)\right]\right.\right.} \\
& \left.-\left[\varepsilon_{\mathrm{HbO}}^{\lambda 1}\left(\Delta O D^{\lambda 2} / B^{\lambda 2}\right)\right]\right\} /\left(\varepsilon_{\mathrm{HbD}}^{\lambda 1} \varepsilon_{\mathrm{HbO}}^{\lambda 2}\right. \\
& \left.\left.-\varepsilon_{\mathrm{HbD}}^{\lambda 2} \varepsilon_{\mathrm{HbO}}^{\lambda 1}\right) L\right], \\
\Delta[\mathrm{HbO}]= & {\left[\left\{\left[\varepsilon_{\mathrm{HbD}}^{\lambda 1}\left(\Delta O D^{\lambda 2} / B^{\lambda 2}\right)\right]\right.\right.} \\
& \left.-\left[\varepsilon_{\mathrm{HbD}}^{\lambda 2}\left(\Delta O D^{\lambda 1} / B^{\lambda 1}\right)\right]\right\} /\left(\varepsilon_{\mathrm{HbD}}^{\lambda 1} \varepsilon_{\mathrm{HbO}}^{\lambda 2}\right. \\
& \left.\left.-\varepsilon_{\mathrm{HbD}}^{\lambda 2} \varepsilon_{\mathrm{HbO}}^{\lambda 1}\right) L\right],
\end{aligned}
$$

where $\lambda_{1}$ and $\lambda_{2}$ are different wavelengths of near-infrared light.

\section{Acknowledgments}

This study was supported in part by NIH Grant No. RO1 DK 068158 (Jadcherla). The authors were grateful to Rebecca Moore, RNC, for nursing observations with this study. This work was performed at Neonatal Nurseries, Nationwide Children's Hospital, Columbus, Ohio. 


\section{References}

1. M. C. Allen, "Neurodevelopmental outcomes of preterm infants," Curr. Opin. Neurol. 21(2), 123-128 (2008).

2. M. Hack, "Survival and neurodevelopmental outcomes of preterm infants," J. Pediatr. Gastroenterol. Nutr. 45(Suppl 3), S141-S142 (2007).

3. B. E. Stephens and B. R. Vohr, "Neurodevelopmental outcome of the premature infant," Pediatr. Clin. North Am. 56(3), 631-646 (2009).

4. M. S. Kramer, "Late preterm birth: appreciable risks, rising incidence," J. Pediatr. 154(2), 159-160 (2009).

5. A. L. van Baar et al., "Functioning at school age of moderately preterm children born at 32 to 36 weeks' gestational age," Pediatrics 124(1), 251-257 (2009).

6. S. Yang, R. W. Platt, and M. S. Kramer, "Variation in child cognitive ability by week of gestation among healthy term births," Am. J. Epidemiol. 171(4), 399-406 (2010).

7. J. Case-Smith, "An efficacy study of occupational therapy with highrisk neonates," Am. J. Occup. Ther. 42(8), 499-506 (1988).

8. S. R. Hintz et al., "Bedside functional imaging of the premature infant brain during passive motor activation," J. Perinat. Med. 29(4), 335-343 (2001).

9. N. Roche-Labarbe et al., "Noninvasive optical measures of CBV, StO (2), CBF index, and $\mathrm{rCMRO}(2)$ in human premature neonates' brains in the first six weeks of life," Hum. Brain Mapp. 31(3), 341-352 (2010).

10. B. R. White et al., "Bedside optical imaging of occipital resting-state functional connectivity in neonates," Neuroimage 59(3), 2529-2538 (2012).

11. J. S. Wyatt et al., "Quantification of cerebral oxygenation and haemodynamics in sick newborn infants by near infrared spectrophotometry," Lancet 328(8515), 1063-1066 (1986).

12. A. D. Edwards et al., "Cotside measurement of cerebral blood flow in ill newborn infants by near infrared spectroscopy," Lancet 332(8614), 770-771 (1988)

13. A. G. Allievi et al., "An fMRI compatible wrist robotic interface to study brain development in neonates," Ann. Biomed. Eng. 41(6), 1181-1192 (2013)

14. T. Arichi et al., "Somatosensory cortical activation identified by functional MRI in preterm and term infants," Neuroimage 49(3), 2063-2071 (2010).

15. D. T. Delpy et al., "Cerebral monitoring in newborn infants by magnetic resonance and near infrared spectroscopy," Scand. J. Clin. Lab. Invest. Suppl. 47, 9-17 (1987).

16. G. Gratton and M. Fabiani, "Shedding light on brain function: the eventrelated optical signal," Trends Cogn. Sci. 5(8), 357-363 (2001).

17. C. Jenny et al., "Reproducibility of cerebral tissue oxygen saturation measurements by near-infrared spectroscopy in newborn infants," J. Biomed. Opt. 16(9), 097004 (2011).

18. A. A. Baird et al., "Frontal lobe activation during object permanence: data from near-infrared spectroscopy," Neuroimage 16(4), 1120-1126 (2002).

19. A. Worley et al., "Multi-modal pain measurements in infants," J. Neurosci. Methods 205(2), 252-257 (2012).

20. S. R. Jadcherla and R. Shaker, "Physiology of aerodigestive reflexes in neonates and adults," in Physiology of the Gastrointestinal Tract, J. Wood and L. Johnson Eds., Elsevier, Amsterdam, Netherlands (2012)

21. M. Pena et al., "Sounds and silence: an optical topography study of language recognition at birth," Proc. Nat. Acad. Sci. U. S. A 100(20), 11702-11705 (2003).

22. V. Quaresima, S. Bisconti, and M. Ferrari, "A brief review on the use of functional near-infrared spectroscopy (fNIRS) for language imaging studies in human newborns and adults," Brain Lang. 121(2), 79-89 (2012).

23. V. Quaresima et al., "Lateral frontal cortex oxygenation changes during translation and language switching revealed by non-invasive nearinfrared multi-point measurements," Brain Res. Bull. 59(3), 235-243 (2002).

24. S. Rossi et al., "Shedding light on words and sentences: near-infrared spectroscopy in language research," Brain Lang. 121(2), 152-163 (2012).

25. D. C. Van Essen, "A population-average, landmark- and surface-based (PALS) atlas of human cerebral cortex," Neuroimage 28(3), 635-662 (2005).
26. J. Hill et al., "A surface-based analysis of hemispheric asymmetries and folding of cerebral cortex in term-born human infants," J. Neurosci. 30(6), 2268-2276 (2010).

27. T. J. Huppert et al., "HomER: a review of time-series analysis methods for near-infrared spectroscopy of the brain," Appl. Opt. 48(10), D280D298 (2009).

28. B. Molavi and G. A. Dumont, "Wavelet-based motion artifact removal for functional near-infrared spectroscopy," Physiol. Meas. 33(2), 259270 (2012).

29. H. Akaike, "A new look at the statistical model identification," IEEE Trans. Autom. Control 19(6), 716-723 (1974).

30. R Development Core Team, "R: a language and environment for statistical computing," R Foundation for Statistical Computing, Vienna, Austria, http://www.R-project.org (2008).

31. C. P. Gaebler and J. R. Hanzlik, "The effects of a prefeeding stimulation program on preterm infants," Am. J. Occup. Ther. 50(3), 184-192 (1996).

32. J. Case-Smith, P. Cooper, and V. Scala, "Feeding efficiency of premature neonates," Am. J. Occup. Ther. 43(4), 245-250 (1989).

33. S. M. Barlow et al., "Synthetic orocutaneous stimulation entrains preterm infants with feeding difficulties to suck," J. Perinatol. 28(8), 541548 (2008).

34. S. Fucile et al., "Oral and nonoral sensorimotor interventions facilitate suck-swallow-respiration functions and their coordination in preterm infants," Early Hum. Dev. 88(6), 345-350 (2012).

35. S. Brigadoi et al., "Motion artifacts in functional near-infrared spectroscopy: a comparison of motion correction techniques applied to real cognitive data," Neurolmage 85(Pt 1), 181-191 (2014)

36. T. Grossmann, E. Parise, and A. D. Friederici, "The detection of communicative signals directed at the self in infant prefrontal cortex," Front. Hum. Neurosci. 4, 201-201 (2010).

37. T. Karen et al., "Hemodynamic response to visual stimulation in newborn infants using functional near-infrared spectroscopy," Hum. Brain Mapp. 29(4), 453-460 (2008).

38. S. M. Liao et al., "Neonatal hemodynamic response to visual cortex activity: high-density near-infrared spectroscopy study," J. Biomed. Opt. 15(2), 026010 (2010).

39. E. Nakato et al., "I know this face: neural activity during mother's face perception in 7- to 8-month-old infants as investigated by near-infrared spectroscopy," Early Hum. Dev. 87(1), 1-7 (2011).

40. N. Naoi et al., "Cerebral responses to infant-directed speech and the effect of talker familiarity," Neuroimage 59(2), 1735-1744 (2012).

41. H. Watanabe, F. Homae, and G. Taga, "Activation and deactivation in response to visual stimulation in the occipital cortex of 6-month-old human infants," Dev. Psychobiol. 54(1), 1-15 (2012).

42. T. Wilcox et al., "Functional activation of the infant cortex during object processing," Neuroimage 62(3), 1833-1840 (2012).

43. J. B. Wagner et al., "Neural processing of repetition and non-repetition grammars in 7- and 9-month-old infants," Front. Psychol. 2, 168-168 (2011).

44. T. Durduran et al., "Optical measurement of cerebral hemodynamics and oxygen metabolism in neonates with congenital heart defects," J. Biomed. Opt. 15(3), 037004 (2010).

45. P. E. Grant et al., "Increased cerebral blood volume and oxygen consumption in neonatal brain injury," J. Cereb. Blood Flow Metab. 29(10), 1704-1713 (2009)

46. S. E. Nicklin et al., "The light still shines, but not that brightly? The current status of perinatal near infrared spectroscopy," Arch. Dis. Child Fetal Neonatal Ed. 88(4), 263F-268 (2003).

47. C. L. Hilton et al., "Review of instrument development and testing studies for children and youth," Am. J. Occup. Ther. 67(3), e30-e54 (2013).

48. Y. Hoshi, "Functional near-infrared spectroscopy: current status and future prospects," J. Biomed. Opt. 12(6), 062106 (2007).

49. G. A. Marimon et al., "Near-infrared spectroscopy cerebral and somatic (renal) oxygen saturation correlation to continuous venous oxygen saturation via intravenous oximetry catheter," J. Crit. Care 27(3), 314.e13314.e18 (2012).

50. E. Vergara et al., "Specialized knowledge and skills for occupational therapy practice in the neonatal intensive care unit," Am. J. Oсcup. Ther. 60(6), 659-668 (2006).

51. R. N. Aslin and J. Mehler, "Near-infrared spectroscopy for functional studies of brain activity in human infants: promise, prospects, and challenges," J. Biomed. Opt. 10(1), 011009 (2005). 
52. G. Pichler et al., "Recommendations to increase the validity and comparability of peripheral measurements by near infrared spectroscopy in neonates. 'Round table', section of haematology, oxygen transport and microcirculation, 48th annual meeting of ESPR, Prague 2007," Neonatology 94(4), 320-322 (2008).

53. C. Mansouri and N. H. Kashou, New window on optical brain imaging: medical development, simulations, applications," in Selected Topics on Optical Fiber Technology, M. Yasin Ed., 270-288, InTech (2011).

Nasser H. Kashou is an assistant professor at Wright State University and an IEEE senior member. He received his $\mathrm{PhD}$ in 2008 from Ohio State University's Biomedical Engineering Department. His education also includes a Master of Science in electrical engineering, 2004. Currently, he is running the Image Analysis Lab (IAL) at Wright State University (WSU). He has also established and runs the functional near infrared spectroscopy lab.

Irfaan A. Dar received his Master of Science in biomedical engineering in 2015 from WSU. Currently, he is continuing his studies as a PhD student at WSU as a member of the IAL and research associate in the Neonatal Infant Feeding Disorders Program at Nationwide Children's Hospital. His expertise includes biomedical signal processing, medical imaging, image processing, near-infrared spectroscopy, and hardware design.
Kathryn A. Hasenstab received her Bachelor of Science degree in biomedical engineering (biomechanics) from the University of Akron. Currently, she is a senior biomedical research associate at the Research Institute at Nationwide Children's Hospital associated with the Neonatal Infant Feeding Disorders and Neonatal Aerodigestive Programs. Her interests include mechanisms of pharyngoesophageal motility, respiration, and gastroesophageal reflux in infants during health and disease.

Ramzi W. Nahhas is highly active in collaborative projects, utilizing modern statistical methods to analyze data from a wide range of biomedical research areas. These disciplines include childhood growth and development, obesity, cardiometabolic risk across the lifespan, illicit drug use, and psychiatry. His primary methodological area of expertise is the analysis of longitudinal data, in particular, linear and nonlinear mixed models.

Sudarshan R. Jadcherla is a professor of pediatrics at the Ohio State University College of Medicine and Public Health. $\mathrm{He}$ is also the principal investigator and director of the Neonatal and Infant Feeding Disorders Research Program at the Nationwide Children's Hospital Research Institute. His research involves understanding the aerodigestive, neurosensory, and neuromotor pathophysiology and functional connectivity of feeding and swallowing related reflexes in human neonates. 